\title{
A Language for Musical Qualia
}

\author{
MARCEL ZENTNER \\ Department of Psychology, University of York, UK
}

\begin{abstract}
In this commentary I build on Phil Barnard's distinction of implicational and propositional meanings as an incentive to explore possibilities for bridging the two types of meanings. I argue that, contrary to the claim that musical experiences are ineffable, the subtle implicational meanings that make up the experiential richness of musical qualia may be amenable to linguistic description and objectification, provided that a suitable language is being used. Taking music-evoked emotional experiences as an example, I sketch the contours of such a language and also discuss criteria by which the suitability of a language to characterize musical qualia may be evaluated.
\end{abstract}

Submitted 2012 January 6; accepted 2012 July 13.

KEYWORDS: meaning, linguistic description, emotive vocabulary, qualia

THE notion of music's effects and meanings as ineffable, since they cannot be communicated, or apprehended by any means other than by direct experience, has a venerable tradition (e.g., Jankélévitch, 2003). This issue is once again brought to the fore by Phil Barnard's article, in which he makes a distinction between implicational and propositional meaning:

The analysis considers two qualitatively different types of meaning originally proposed by Barnard and Teasdale (1991) to analyse relationships between cognition, affect and embodiment. One form of meaning relates to the encoding of "propositions," whilst the other blends ideas, externally derived percepts and bodily experience to form "implicational meanings." These are more abstract schemata or models of deep multimodal interdependences that equate more with latent senses of meaning or knowing as well as feelings, intuitions and affect. Propositional and implicational meanings co-exist in mental architecture and depend one upon the other. While language skills are more clearly focused on the form and expression of propositional meanings, musical expertise and aesthetic appreciation links most readily to implicational meanings.

Barnard further notes that "forms like poetry, metaphor and parable more accurately capture the rich yet latent high dimensionality of implicational meanings." For the sake of brevity, I will not scrutinize this distinction any further. Rather, I shall take it as a point of departure for discussing a question, long known in the philosophy of consciousness, namely, whether experiences of implicational meaning can be, if not comprehended entirely, at least indexed by means of some form of language.

The implication of this distinction is not that music scholars would generally engage in a pointless initiative, due to the absence of a system for translating implicational meaning into propositional meaning. Indeed, it is possible to analyze the characteristics of various musical systems, describe how people relate to music across different contexts and cultures, describe music education programs at different levels, or report findings on brain correlates of musical processing and so on. Such works speak of music, and do so in meaningful ways. Thus, the notion of ineffability does not relate to any form of proposition about music. What seems to elude a transcription into propositional terms is the inner experience of music, the "what-it-is-like-to-experience-music." It is the apparent impossibility to characterize the experience of music that has earned this experience the reputation of being unspeakable. In what may seem a strange opening to a book, Daniel Barenboim notes: "I firmly believe that it is impossible to speak about music" (Barenboim, 2008, p. 5).

Because there is a relatively broad consensus that musical experiences are often of an emotive kind (Juslin \& Sloboda, 2010), it is worthwhile to briefly examine how research on emotion has dealt with the problem of objectifying a subjective inner experience. Mindful of the difficulties in capturing the rich implicational meanings of the experience of emotion, emotion psychologists have prioritized the analysis and description of expressions of emotion, in particular those overt ones that can be measured. These measurable or quantifiable "exteriorizations" include the physiological correlates of emotion, the expression of emotion in face and voice, the action tendencies associated with emotions, and the verbal reports of emotional feelings. 
The copious literature describing these relationships carries two important messages. First, although the inner experience of an emotion is a private and subjective one, its expressions are amenable to scientific description, quantification, and analysis. This has led emotion psychologists to look at the similarity or dissimilarity of individuals' emotive reactions to specific situations based on how their emotions are expressed. For example, the reaction to an emotion trigger, say, the sudden sight of a snake, is systematically followed by indicators of physiological arousal (e.g., amygdala activation in the brain, heart rate acceleration), action tendencies (startle, followed by turning away and flight), and facial expressions characteristic of fear that are similar across people. Thus, although we cannot know what people's inner experience to the sight of a snake might feel like, the assumption is that the similarity in emotion expression is subtended by an interpersonally similar inner experience of the emotion. In other words, the similarity and dissimilarity of inner subjective experiences across individuals, while not directly accessible, can nonetheless be inferred.

This would seem to suggest that the notion that because experiences are subjective and private, all interpersonal comparisons of inner experiences are systematically impossible (e.g., Dennett, 1991) is exaggerated. Indeed, why should we not be able to gauge the extent of similarity in listeners' inner experiences to a piece of music by relying on the similarity with which these experiences are being expressed? What seems impossible is only a direct characterization of the inner experience, what is referred to as "quale" in the philosophy of consciousness. Qualia, the plural of quale, is a term used to refer to subjective conscious experiences as "raw feels" (Ferber, 2003). A frequently cited description of the nature of qualia is a thought experiment by Jackson (1982), which runs as follows:

Mary the colour scientist knows all the physical facts about colour, including every physical fact about the experience of colour in other people, from the behavior that the particular colour is likely to elicit to the specific sequence of neurological firings that register that a colour has been seen. However, she has been confined from birth to a room that is black and white, and is only allowed to observe the outside world through a black and white monitor. When she is allowed to leave the room, it must be admitted that she learns something about the colour red the first time she sees it specifically, she learns what it is like to see that colour.

Many responses and criticisms have been offered in response to this thought experiment (e.g., Tye, 2009; Wright, 2008). Our question here is whether there is a form of knowledge that might have made Mary's experience of red, when she finally sees it, less novel or surprising than it might have otherwise been. Since qualia seem easier to communicate through poetry, metaphor, analogy, and parable (Lakoff \& Johnson, 1999), we ought to ask how much "metaphoric knowledge" of red Mary had. We may contend that, by having read extensively about poetic and metaphoric characterizations of red, Mary might still have learned something new about the color red when seeing it, but she might have been less surprised or intrigued by the experience.

This scenario raises the possibility of a language at the interface of science and poetry that may serve as a better approximation to qualia than is offered by declarative or propositional statements and yet be amenable to formalization and quantification. Such a language does not currently exist, either generically or for the special case of musical qualia. Perhaps contrary to expectation, it is not the development of such a language that presents the greatest challenge. The real difficulty is to set up criteria against which the merits or advantages of a vocabulary or language over other possible vocabularies or languages can be systematically evaluated. Because this may sound somewhat abstract, I will briefly describe research conducted in our laboratory that illustrates this point. In our work on the characterization and classification of music-induced emotion, we first devised a vocabulary to describe the emotive characteristics of musical experiences. The method for creating this vocabulary is described elsewhere, but essentially we started out with 515 affect terms that were progressively narrowed down to those that repeated experiments had shown to be most useful in describing musicinduced emotion. This resulted in the Geneva Emotional Music Scale, also called GEMS (Zentner, Grandjean, \& Scherer, 2008; Zentner, 2010). The GEMS vocabulary, though not a metaphoric vocabulary proper, consists nevertheless of terms that would be more typically used by novelists than by experimental psychologists or philosophers, including such terms as ardent (fiery), bouleversé (overwhelmed), emerveillé (filled with wonder), ému (moved), héroïque (heroic), rêveur (dreamy), sautillant (bouncy), and sentiment de transcendance (feeling of transcendence).

Once developed, we tested the merits of the GEMS against alternative emotion vocabularies that had been used previously to describe the emotive properties of musical experiences. The incremental validity of the GEMS vocabulary over alternative descriptive schemes was evaluated on the basis of three criteria: (a) phenomenological plausibility; (b) sensitivity in capturing interpersonal similarity in emotive experience, and (c) power of discriminating musical excerpts based on affect ratings. The first criterion is met when listeners more frequently choose to describe their affective reactions to music with terms provided by our vocabulary than with terms derived from alternative vocabularies. Second, only a vocabulary suited to capture the emotive features of musical qualia can identify interpersonal similarity in musical experience, when it exists. A deficient vocabulary more 
likely leads to arbitrary responses and thus an absence of agreement. Consider a situation in which people have four choices to describe a Prussian blue: black, grey, brown, and purple. Because these are about equally imperfect descriptors of the color blue, individuals would likely differ in their choices, leading to a heterogeneous response pattern. If, however, the selections were navy, black, brown, and purple, most individuals would probably converge on navy as the closest approximation to the Prussian blue. In other words, the higher the coefficients of intersubjective agreement (that can be indexed with coefficients such as Cronbach's $\alpha$, McDonald's $\omega$ or the intraclass coefficient, depending on the nature of the data), the more likely it is that the language has been capturing some essential features the musical qualia.

The last criterion relates to a form of reverse engineering. We asked how well various musical excerpts could be differentiated and classified from the GEMS compared to the classification obtained from alternative descriptive vocabularies for emotion. An apt vocabulary for describing musical qualia should be able to "return" the stylistic, rhythmic, melodic, timbric, and harmonic aspects of musical pieces in a more nuanced way than an inept vocabulary. Thus, while the latter may manage to group musical works into two categories such as slow and fast ones, the former might be able to return the distinction between pieces from the classical vs romantic period, or between hip-hop vs hard rock songs. Methods such as cluster analysis can be used to examine the granularity and accuracy of such groupings. There is also a nonlinguistic alternative to probe the third criterion. Individuals can make similarity judgments of musical experiences without using any verbal labels. In such experiments, participants can be required to listen to various pieces and to group together those that evoked similar (emotional) experiences. Multidimensional scaling techniques can be used to examine whether the nonverbal groupings converge with the classification obtained from verbal reports. The eventual presence of such convergence would denote "cross-validation," that is, the confirmation of a finding by at least two independent assay techniques. As is described in our publications (Trost, Ethofer, Zentner, \& Vuilleumier, 2012; Zentner et al., 2008; Zentner, 2010), our vocabulary fulfilled these three criteria to some extent, thereby increasing our confidence in having identified a language that has the potential to characterize and classify emotive properties of music-evoked qualia.

To recapitulate, I have used (or, rather, misused) Phil Barnard's distinction of implicational and propositional meanings as an incentive to explore possibilities for bridging the two types of meanings. I have argued that, rather than being hopelessly ineffable, the subtle implicational meanings that make up the experiential richness of musical qualia may be amenable to linguistic description, albeit of a special kind that warrants further study and development.

\section{ACKNOWLEDGMENTS}

I wish to thank Professor Rafael Ferber for his comments on the philosophical parts of this commentary.

\section{REFERENCES}

Barenboim, D. (2008). Everything is connected. The power of music. London, England: Weidenfeld \& Nicolson.

Dennett, D. C. (1991). Consciousness explained. London, England: Allen Lane.

Ferber, R. (2003). Philosophische Grundbegriffe: Vol. 2. Munich, Germany: Beck.

Jackson, F. (1982). Epiphenomenal qualia. Philosophical Quarterly, Vol. 32, pp. 127-136.

Jankélévitch, V. (2003). Music and the ineffable. Princeton, NJ: Princeton University Press.

Juslin, P., \& Sloboda, J. (2010). Music and emotion. Oxford, UK: Oxford University Press.

Lakoff, G., \& Johnson, M. (1999). Philosophy in the flesh: The embodied mind and its challenge to Western thought. New York, NY: Basic Books.

Trost, W., Ethofer, T., Zentner, M., \& Vuilleumier, P. (2012, in press). Mapping aesthetic musical emotions in the brain. Cerebral Cortex. 
Tye, M. (2009). Consciousness revisited. Materialism without phenomenal concepts. Cambridge, MA: MIT Press.

Wright, E. (2008). The case for qualia. Cambridge, MA: MIT Press.

Zentner, M., Grandjean, D., \& Scherer, K. R. (2008). Emotions evoked by the sound of music: Characterization, classification and measurement. Emotion, Vol. 8, pp. 494-521.

Zentner, M. (2010). Homer's prophecy: An essay on music's primary emotions. Music Analysis, Vol. 29, pp. 102-125. 